\title{
Coarse graining of slow variables in dynamic simulations of soft matter
}

\author{
A. van den Noort, W. K. Den Otter and W. J. Briels ${ }^{(a)}$ \\ Computational Biophysics, Faculty of Science and Technology, University of Twente \\ P.O. Box 217, 7500 AE Enschede, The Netherlands
}

received 23 March 2007; accepted in final form 21 August 2007

published online 14 September 2007

PACS 83.10.Mj- Molecular dynamics, Brownian dynamics

PACS 83.60.Rs - Shear rate-dependent structure (shear thinning and shear thickening)

PACS 83.80.Hj-Suspensions, dispersions, pastes, slurries, colloids

\begin{abstract}
A new Brownian dynamics model is presented to describe the coarse grain dynamics of particles with long-lived memory. Instead of solving a set of generalized Langevin equations we introduce a set of variables describing the slowly fluctuating thermodynamic state of the ignored degrees of freedom. These variables give rise to additional transient forces on the simulated particles, whose interpretation provides a new way of thinking about memory effects in soft-matter physics. We illustrate the proposed method by simulating shear thinning of synthetic resins.
\end{abstract}

Copyright (c) EPLA, 2007

In many instances in soft-matter physics it is sufficient to restrict attention to a small number of variables out of a much larger set describing the system in full detail. An example is provided by a system composed of $N$ colloids floating in a solvent in which possibly a small amount of polymer is dissolved [1-4]. Experimental studies [5] and simulations of this system usually address only the $N$ position vectors $r^{3 N}=\left\{\mathbf{r}_{1}, \ldots, \mathbf{r}_{N}\right\}$ of the colloids. Similarly in a solution or a melt of star polymers [6,7] many phenomena can be understood by studying the position vectors of the centres of mass of the stars, ignoring all other degrees of freedom. Even with melts of linear polymers, it may be sufficient to restrict attention to the centres of mass of all molecules [8]. Obviously, many detailed questions cannot be answered with the restricted knowledge of the trajectories $r^{3 N}(t)$ alone, but it is well known that all thermodynamic and rheological properties can be calculated, provided the correct interactions between the coarse particles are used, and the correct propagator is used to calculate the trajectory. The correct interactions derive from the appropriate [9] free energy $\Phi\left(r^{3 N}\right)$, of the ignored coordinates at the given configuration $r^{3 N}$ of the simulated coordinates. Much progress has been made with finding accurate pairwise additive approximations for $\Phi\left(r^{3 N}\right)$, which can profitably be used in both theory and simulations [10-12]. The correct propagator is the generalized Langevin propagator [13-15] with

${ }^{(a)}$ E-mail: w.j.briels@utwente.nl potential forces deriving from $\Phi\left(r^{3 N}\right)$ and with friction forces and corresponding random forces governed by twoparticle time-dependent friction kernels $\boldsymbol{\zeta}_{i j}(t)$ to represent the coupling of the simulated coordinates to the "bath of ignored coordinates". Only in cases when the dynamics of the simulated coordinates is much slower than that of the bath, much progress has been made. In all examples given above, however, this condition is not met and severe problems occur with existing methods. In this letter we describe a method which solves this problem in a number of cases.

Before presenting the details of our method, a few more comments on existing methods to simulate the dynamics of soft matter systems must be made. First, assuming complete separation of time scales between the ignored and simulated degrees of freedom, the friction kernels may be taken to be proportional to the Dirac $\delta(t)$. This turns the generalized Langevin equations of motion into the usual Langevin equations, as used for example in the well-known DPD method [16-18]. Second, in most examples of soft matter the frictions are large enough to make all velocities relax to equilibrium long before the corresponding coordinates have changed appreciably. In such cases all velocities may be eliminated $[19,20]$ from the description and the coordinates are found to move according to a set of first-order differential equations, generically called Brownian dynamics equations [21]. In the particular case of colloids dissolved in Newtonian liquids the mobilities, i.e. the inverse friction tensors, 


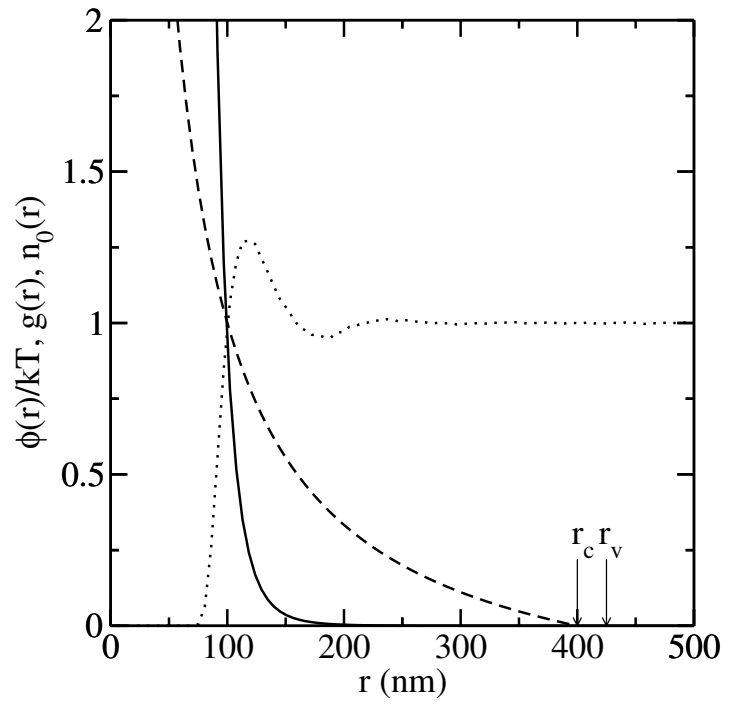

Fig. 1: The pair potential $\phi(r)$ (solid line), radial distribution function $g(r)$ (dotted line) and equilibrium number of stickers $n_{0}(r)$ (dashed line).

may explicitly be calculated assuming Stokes flow of the solvent at the given configurations of the colloids, in which case the method is called Stokesian dynamics [22,23]. In other cases more ad hoc assumptions must be made. To the best of our knowledge no successful Brownian dynamics method including memory effects has been suggested so far. It is this problem that we address in this letter. For ease of presentation we apply our method to the specific case describing the rheology of synthetic resins. At the end of this letter we will mention other problems to which it may successfully be applied.

Synthetic waterborne resins to be used in paints, coatings and varnishes, are complex systems often consisting of hard-core particles densely coated with a thick corona of long, charged and/or neutral chains, dissolved in water containing many oligomeric and polymeric components $[24,25]$. Hydrophobic and hydrophilic groups along these chains act as weak "stickers" between the coronas. Hard-core diameters range from 75 to $150 \mathrm{~nm}$ and volume fractions of hard material are about $20 \%$. Effective pair potentials representing the free energy of the polymer arms at the given configuration of the centres of mass of the particles are very soft at pair distances beyond the hard-core diameter, resulting in easily penetrable soft spheres with small hard pits. As a potential having these characteristics we have chosen $\phi(r)=4 \epsilon\left(\frac{b}{r}\right)^{8}$. The values of $\epsilon$ and $b$ are dictated by the specific particle that is to be simulated, here a particle with hard-core diameter $\sigma=100 \mathrm{~nm}$ and a soft repulsive potential beyond this value. In all our calculations we have used $\epsilon=240 k_{B} T$, $T=300 \mathrm{~K}$ and $b=42 \mathrm{~nm}$. This potential is drawn in fig. 1 . With decreasing values of $r$ the potential steeply rises near $r=100 \mathrm{~nm}$. Several criteria have been developed [26] to associate a hard-core diameter $\sigma$ with a potential like this, all resulting in about the same value. Here we adopt the rule $\phi(\sigma)=k_{B} T$. This results in a hard-core diameter of $\sigma=100 \mathrm{~nm}$. The soft repulsive tail quickly decays and is equal to zero for all practical purposes at the cut-off distance $r_{c}=4 \sigma$. The latter value is the diameter of the full particle, including the corona.

At equilibrium, the probability density of the coordinates $r^{3 N}$ is given by

$$
\begin{gathered}
\Psi\left(r^{3 N}\right) \propto \exp \left\{-\beta \Phi\left(r^{3 N}\right)\right\}, \\
\Phi(r)=\sum_{\langle i, j\rangle} \phi\left(r_{i j}\right),
\end{gathered}
$$

with $\beta=\left(k_{B} T\right)^{-1} . \Phi\left(r^{3 N}\right)$ is the free energy of all ignored coordinates at the given configuration $r^{3 N}$ of the centres of mass. It is well defined in all practical cases, but is usually difficult to represent. It is not our aim, in this paper, to study this problem. The representation given in the second line of eq. (1) is only chosen to have an easy, but realistic model to perform simulations. For clarity of presentation, and without loss of generality, it may be assumed to be exact. In fig. 1 is shown the radial distribution function resulting from this potential at a number density $\rho=400 \mu \mathrm{m}^{-3}$. It reaches unity for the first time at $r=100 \mathrm{~nm}$, corroborating the earlier choice of $\sigma=100 \mathrm{~nm}$. This results in a volume fraction of hard material $\varphi=0.2$ for all simulations described in this letter.

The dynamics of the coronas is expected to be equally slow as the conformational changes of the centres of mass of the particles. Besides the centre-of-mass positions $r^{3 N}(t)$ we therefore propose to retain one degree of freedom for each corona. Their time evolution represents the relaxation back to equilibrium of all coronas after the configuration of the centres of mass has been changed. We call $n_{i}$ the number of stickers of particle $i$ with its direct neighbours. Its value may range from zero to some maximum $n_{\max }$. We want to stress that the name "sticker" should not be taken too literally. Besides stickers, also entanglements between polymer arms are characteristic for the state of affairs of the coronas. We calculate the number of stickers of particle $i$ as $n_{i}=\sum_{j} n_{i j}$, where $n_{i j}$ is the number of stickers between particles $i$ and $j$. We assume furthermore that for a distance $r_{i j}$ between the particles $i$ and $j$ the number of stickers $n_{i j}$ on average is equal to $n_{0}\left(r_{i j}\right)$. At equilibrium, the joint probability density of the coordinates $r^{3 N}$ and stickers $n^{N}$ is taken to be

$$
\begin{gathered}
\Psi\left(r^{3 N}, n^{N}\right) \propto \exp \left\{-\beta\left[\Phi\left(r^{3 N}\right)+\sum_{\langle i, j\rangle} \frac{1}{2} \alpha\left(n_{i j}-n_{0}\left(r_{i j}\right)\right)^{2}\right]\right\} \\
n_{0}(r)=1-\frac{r_{c}}{r_{c}-\sigma} \frac{r-\sigma}{r}
\end{gathered}
$$

Accepting negative values of $n_{i j}$, an integration over all $n_{i j}$ proves that the coordinates are distributed according to the exact Boltzmann distribution $\propto \exp \left\{-\beta \Phi\left(r^{3 N}\right)\right\}$. The introduction of sticker numbers therefore does not 
destroy the exact structural properties of the centres of mass of the particles, nor the exact thermodynamic properties of the system $[11,15,27]$. The parameter $\alpha$ occurring in eq. (2) fixes the allowed fluctuations of the $n_{i j}$. The equilibrium number of stickers $n_{0}(r)$, dashed line in fig. 1, should be roughly proportional to the overlap volume of two spheres of diameter $r_{c}$, separated by a distance $r$. The precise functional form of $n_{0}(r)$ was chosen on the basis of convenience. Moreover, $n_{0}(r)$ was chosen to be unity at $r=\sigma$, since it can be scaled to any value by an appropriate change of $\alpha$. From information about the synthesis of the experimental particle we inferred $r_{c}=4 \sigma$. The rather large value of $r_{c}$ compared to the range of the pair potential $\phi(r)$ reflects the fact that the size of the corona is much larger than the size of the hard core. Of course, depending on the precise application, other forms for $n_{0}(r)$ may turn out to be more appropriate.

The Brownian dynamics propagator admitting the above probability density as a stationary distribution may be derived using the standard procedure for obtaining the Smoluchowski equation [18-20], and reads

$$
\begin{aligned}
\mathrm{d} \mathbf{r}_{i}= & \left\langle\mathbf{V}\left(\mathbf{r}_{i}\right)\right\rangle \mathrm{d} t+\nabla_{i}\left(k_{B} T / \xi_{i}\right) \mathrm{d} t+\boldsymbol{\theta} \sqrt{2 k_{B} T \mathrm{~d} t / \xi_{i}} \\
& +\frac{1}{\xi_{i}} \sum_{j}\left\{-\nabla_{i} \phi\left(r_{i j}\right)+\alpha\left(n_{i j}-n_{0}\left(r_{i j}\right)\right) \frac{\mathrm{d} n_{0}}{\mathrm{~d} r_{i j}} \frac{\mathbf{r}_{i j}}{r_{i j}}\right\} \mathrm{d} t, \\
\mathrm{~d} n_{i j}= & -\frac{1}{\tau}\left(n_{i j}-n_{0}\left(r_{i j}\right)\right) \mathrm{d} t+\theta^{\prime} \sqrt{2 k_{B} T \mathrm{~d} t / \alpha \tau} .
\end{aligned}
$$

Here $\left\langle\mathbf{V}\left(\mathbf{r}_{i}\right)\right\rangle$ is the average flow field at position $\mathbf{r}_{i}$ and $\boldsymbol{\theta}$ and $\theta^{\prime}$ are random numbers with variance unity. There are three points to be discussed. First, $\xi_{i}$ is the friction coefficient for the motion of particle $i$ relative to the average local velocity $\left\langle\mathbf{V}\left(\mathbf{r}_{i}\right)\right\rangle$. It consists of a constant term $\xi_{0}$ describing the friction with the solvent and a term describing the friction with the surrounding fellow particles. The latter, for each partner $j$, is proportional to the number of stickers between $i$ and $j$, if this is positive, and zero otherwise. $\xi_{i}$ may therefore be written as

$$
\xi_{i}=\xi_{0}+\frac{1}{2} \xi_{e} \sum_{j}\left(n_{i j}+\left|n_{i j}\right|\right) n_{0}\left(r_{i j}\right) .
$$

The factor $n_{0}\left(r_{i j}\right)$ has been added to make sure that no friction occurs with partners beyond the cut-off distance $r_{c}$. We call $\xi_{e}$ the friction per sticker. Second, $\tau$ is a characteristic time for the rapidity with which the sticker number strives towards its equilibrium value $n_{0}\left(r_{i j}\right)$ for $r_{i j}<r_{c}$ and zero for $r_{i j} \geqslant r_{c}$. Third, we only keep track of $n_{i j}$ for those pairs which are separated by a distance $r_{i j}$ smaller than some value $r_{v}$, slightly larger than $r_{c}$, $r_{v}=1.0625 r_{c}$. When a particular pair enters this region, its sticker number $n_{i j}$ is initialized according to the probability density $\propto \exp \left\{-\frac{1}{2} \beta \alpha n_{i j}^{2}\right\} \cdot r_{v}$ must be slightly larger than $r_{c}$ because, when two separating particles reach a distance $r_{c}$, their sticker number may well be substantially different from zero, and should be given some time to relax to zero in order to prevent the

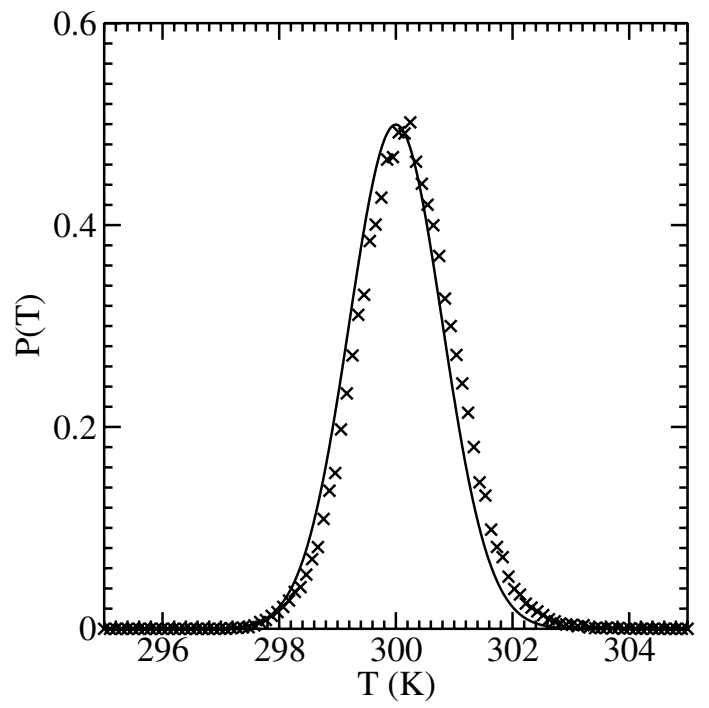

Fig. 2: Temperature distribution based on fluctuations of the sticker number. The solid line shows the theoretical distribution and the symbols give the distribution obtained from the simulation.

occurrence of discontinuities in the sticker forces at $r=r_{c}$. We have checked that with this procedure changing $\alpha$ does not lead to any changes in the radial distribution function of eq. (1).

It is perhaps illuminating to notice that the first member of eq. (3) may be interpreted as expressing a balance of all forces acting on particle $i$. Dividing through by $\mathrm{d} t$, multiplying by $\xi$, and bringing the term on the left-hand side to the first position on the right-hand side, leaves us with six terms which add up to zero. The first two of these represent the friction, the next two the Brownian force, and the last two the potential force. The average velocity $\left\langle\mathbf{V}\left(\mathbf{r}_{i}\right)\right\rangle$ is calculated as the average displacement $\mathrm{d} \mathbf{r}_{j}$ of all particles $j$ near $\mathbf{r}_{i}$, divided by $\mathrm{d} t$. All simulations were done at the state point $\varphi=0.2$ and $T=300 \mathrm{~K}$, as already mentioned, and the model parameters $\alpha=0.473 k_{B} T, \tau=20 \mathrm{~s}, \xi_{0} / \rho k_{B} T=0.603 \mu \mathrm{m}$ $s=0.302 \sigma \tau$ and $\xi_{e} / \rho k_{B} T=2.361 \mu \mathrm{m} \quad s=1.181 \sigma \tau$. Of these, $\xi_{0}$ was measured experimentally at low concentrations. So, three parameters are left to be adjusted to the experimental results. Of these, $\tau$ is fixed by the shear rate where shear thinning starts, $\alpha$ roughly determines the slope in the shear thinning region, while $\alpha$ and $\xi_{e}$ together determine the height of the flow curve. It is important to realize that $T$ is the temperature imposed by the propagator eq. (3). We measured the actual temperature $T_{C}$, defined by $\left\langle\sum \nabla_{i} \Phi \cdot \nabla_{i} \Phi\right\rangle=k_{B} T_{C}\left\langle\sum \nabla_{i}^{2} \Phi\right\rangle[28-30]$ and found it to settle to a value of $303 \mathrm{~K}$ after an equilibration time of a few $\tau$. In fig. 2 we plot the distribution of temperatures $T_{E}$ defined by $\sum\left\langle\alpha\left(n_{i j}-n_{0}\left(r_{i j}\right)\right)^{2}\right\rangle=N_{P} k_{B} T_{E}$, where the sum runs over all pairs with $r_{i j}<r_{v}$ and $N_{P}$ is the average number of such pairs. The results are seen to be in good agreement with the exact analytical prediction (drawn line) [31], except for a displacement by a tiny 


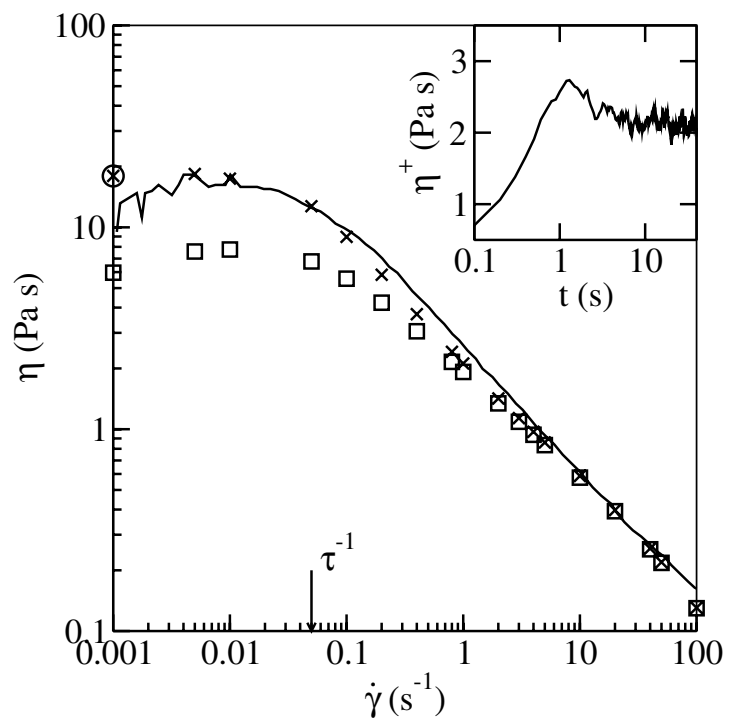

Fig. 3: Viscosity vs. shear rate for a typical resin: the solid line represents experimental data [25], while the crosses are simulation results including all forces in the stress tensor. The squares represent viscosities based on the conservative forces only. The circle at the vertical axis gives the zero-shear viscosity according to the Green-Kubo formalism. The overshoot in the instantaneous viscosity $\eta^{+}(t)$ is plotted as an inset for $\dot{\gamma}=1 \mathrm{~s}^{-1}$.

$0.2 \mathrm{~K}$. A similar displacement occurs in the distribution of temperatures with the DPD model as a result of the rather simple integrator used with stochastic differential equations [32]. The fact that both $T_{C}$ and $T_{E}$ are equal to the input value $T$ proves that the fluctuation-dissipation balance is implemented appropriately.

We measured viscosities by means of non-equilibrium simulations using Lees-Edwards boundary conditions [33] to impose a flow in the $x$-direction with a velocity gradient $\dot{\gamma}$ in the $y$-direction. Stresses were calculated as $\boldsymbol{\sigma}=$ $\frac{1}{V} \sum \mathbf{r}_{i} \mathbf{F}_{i}$ with $\mathbf{F}_{i}$ the total force on particle $i$, i.e. the sum between curly brackets in the first line of eq. (3), and $V$ the volume of the box. Viscosities then followed from $\eta=\sigma_{x y} / \dot{\gamma}$.

In fig. 3 are plotted the experimental viscosities (drawn line) [25] of a typical resin with hard core diameter of $100 \mathrm{~nm}$. Very similar curves are found with other core-shell particles [34]. Also given in this figure are the results of our simulations (crosses). The circle on the vertical axis results from an equilibrium simulation using the Green-Kubo formalism. The squares represent the contribution to the viscosity of the potential forces $-\nabla_{i} \phi\left(r_{i j}\right)$. The remaining part stems from the sticker forces $\alpha\left(n_{i j}-n_{0}\left(r_{i j}\right)\right) \frac{\mathrm{d} n_{0}\left(r_{i j}\right)}{\mathrm{d} r_{i j}}$ directed along $\mathbf{r}_{i j}=\mathbf{r}_{i}-\mathbf{r}_{j}$, and is seen to constitute about two-thirds of the total viscosity at the lower shear rates. For shear rates larger than $1 / \tau$ the sticker kinetics is too slow to appreciably change the number of stickers during the time needed by the flow to separate two particles. As a result the contribution of the sticker forces to the stress becomes constant and consequently their contribution to the viscosity gradually drops to zero. The inset in fig. 3 shows $\eta^{+}(t)=\sigma_{x y}(t) / \dot{\gamma}$, the viscosity after the onset of shear, for a shear rate of $1 \mathrm{~s}^{-1}$. The overshoot near $t=1 \mathrm{~s}$ is completely due to the sticker forces, with no contribution from the potential forces. Since initially the sticker distribution is at equilibrium, large forces are needed to deform the system. Only after a strain of about one has been obtained, the stationary state sets in with stresses corresponding to the imposed shear rate. The same phenomenon is found in experimental curves at about the same time, or better the same strain, but rather more pronounced. Before striving for quantitative agreement more information is needed about $\phi(r)$ beyond the hard-core diameter.

We now summarize the characteristics of our model. It describes the time evolution of the configurations of a set of "particles" in a slow "bath" of ignored coordinates. Besides the direct forces between the particles each of them experiences three types of forces, mediated by the bath. In very rough terms one may say that the propagator eq. (3) results from a balance of all these forces. First there are friction forces exerted by the bath on the particles. Second there are potential forces deriving from the free energy of the ignored coordinates at the given configuration of the simulated coordinates, i.e. the particles. They describe the forces felt by the particles, after the bath of ignored coordinates has relaxed to equilibrium at the given configuration of the particles. Deviations of the bath from equilibrium are described by a set of parameters, $n_{i j}-n_{0}\left(r_{i j}\right)$ in our case, which relax to zero with a characteristic time $\tau$. As long as the bath is not at equilibrium each particle $i$ experiences a third force $-\nabla_{i}\left(\sum \frac{1}{2} \alpha\left(n_{i j}-n_{0}\left(r_{i j}\right)^{2}\right)\right.$. If a pair of particles is brought together such that temporarily $n_{i j}<n_{0}\left(r_{i j}\right)$ the coronas push each other apart leading to a repulsion felt by the particles. If, after $n_{i j}$ has relaxed to $n_{0}\left(r_{i j}\right)$, the particles are separated again such that temporarily $n_{i j}>n_{0}\left(r_{i j}\right)$ the particles will experience attractive forces as long as the bath has not yet relaxed to equilibrium. It is these transient forces, providing memory to the particles, which constitute the novelty of our method. Incorporation of these forces extends the applicability of Brownian dynamics simulations to systems for which traditional Brownian dynamics simulations with deltacorrelated random displacements fail. In cases where memory effects do not play a role, other methods, like for example Stokesian dynamics, may be a much better choice.

Memory effects as described in the previous section occur frequently in soft matter physics, and the model that we have presented in this letter may find many applications, either with or without small adjustments. As a direct application we have recently successfully studied shear banding and the chaining of dissolved colloids in visco-elastic systems [35]. Only small adjustments are needed to apply the model to describe the rheology of linear polymer melts by simulating each polymer as one 
particle [36]. A very interesting problem to tackle with our approach is the inversion in phase separating dynamically inhomogeneous systems. Interesting applications may also be found in the rheology and slow dynamics of polymer blends and block co-polymers.

\section{$* * *$}

This work is supported with a grant of the Dutch Programme EET (Economy, Ecology, Technology), a joint initiative of the Ministeries of Economic Affairs, Education, Culture and Sciences and of Housing, Spatial Planning and the Environment.

\section{REFERENCES}

[1] VRiJ A., Pure Appl. Chem, 48 (1976) 471.

[2] Lekkerkerker H. N. W., Poon W. C. K., Pusey P. N., Stroobants A. and Warren P. B., Europhys. Lett., 20 (1992) 559.

[3] Tuinier R., Vliegenthart G. A. and Lekkerkerker H. N. W., J. Chem. Phys., 113 (2000) 10768.

[4] Anderson V. J. and LekKerkerker H. N. W., Nature, 416 (2002) 811.

[5] Segre P. N., Meeker S. P., Pusey P. N. and Poon W. C. K., Phys. Rev. Lett., 75 (1995) 958.

[6] Vlassopoulos D., Fytas G., Pakula T. and Roovers J., J. Phys.: Condens. Matter, 13 (2001) R855.

[7] Watanabe H., Matsumiya Y., Ishida S., Takigawa T., Yamamoto T., Vlassopoulos D. and Roovers J., Macromolecules, 38 (2005) 7404.

[8] Murat M. and Kremer K., J. Chem. Phys., 108 (1998) 4340.

[9] Dijkstra M., van Roij R. and Evans R., Phys. Rev. E, 59 (1999) 5744.

[10] Likos C. N., Phys. Rep. R, 348 (2001) 267.

[11] Louis A. A., J. Phys.: Condens. Matter, 14 (2002) 9187.

[12] Likos C. N., Soft Matter, 2 (2006) 478.

[13] Deutch J. M. and Oppenheim I., J. Chem. Phys., 54 (1971) 3547.

[14] Murphy T. J. and Aguirre J. L., J. Chem. Phys., 57 (1972) 2098.

[15] Akmermans R. L. C. and Briels W. J., J. Chem. Phys., 113 (2000) 6409.
[16] Hoogerbrugge P. J. and Koelman J. M. V. A., Europhys. Lett., 19 (1992) 155.

[17] Koelman J. M. V. A. and Hoogerbrugge P. J., Europhys. Lett., 21 (1993) 363.

[18] Espanol P. and Warren P., Europhys. Lett., 30 (1995) 191.

[19] Gardiner C. W., Handbook of Stochastic Methods (Springer, Berlin) 1985.

[20] Briels W. J., Theory of Polymer Dynamics, Lect. Notes (Uppsala University) 1994.

[21] Heyes D. M. and Melrose J. R., J. Non-Newtonian Fluid Mech., 46 (1993) 1.

[22] Brady J. F. and Bossis G., Annu. Rev. Fluid Mech., 20 (1988) 111.

[23] Foss D. R. and Brady J. F., J. Fluid Mech., 407 (2000) 167.

[24] Ballauff M., Macromol. Chem. Phys., 204 (2003) 220.

[25] Schellekens H., private communication, Nuplex Resins, Bergen op Zoom, The Netherlands.

[26] Hansen J.-P. and McDonald I. R., Theory of Simple Liquids (Academic Press, London) 1986.

[27] Briels W. J. and Aknermans R. L. C., Mol. Simul., 28 (2002) 145.

[28] Rugh H. H., Phys. Rev. Lett., 78 (1997) 772.

[29] Butler B. D., Ayton G., Jepps O. G. and Evans D. J., J. Chem. Phys., 109 (1998) 6519.

[30] Allen M. P., Ensembles and Averages, Lect. Notes (CCP5 Summer School) 1998.

[31] Frenkel D. and Smit B., Understanding Molecular Simulation (Academic Press, San Diego) 1996.

[32] den Otter W. K. and Clarke J. H. R., Europhys. Lett., 53 (2001) 426.

[33] Allen M. P. and Tildesley D. J., Computer Simulation of Liquids (Clarendon, Oxford) 1987.

[34] Nakamura H. and TACHI K., Colloid Interface Sci., 297 (2006) 312.

[35] VAN DEN NoORT A. and BRIEls W. J., Coarse grained simulations of elongational viscosities, superposition rheology and shear banding in model core-shell systems, to be published in Macromol. Theory Simul. (2007); Simulations of string formations of colloids in a shear thinning medium, in preparation (2007).

[36] Kindt P. and Briels W. J., A single particle model to simulate the dynamics of entangled polymer melts, to be published in J. Chem. Phys. (2007). 